\title{
Neue Sensorentwicklungen für das Brauwesen
}

\author{
S. Päßler ${ }^{1}$, U. Enseleit ${ }^{1}$, W. Oelßner ${ }^{1}$, W. Vonau ${ }^{1}$, , A. Bockisch ${ }^{2}$, S. Junne ${ }^{2}$, P. Neubauer ${ }^{2}$ J. Henze ${ }^{3}$ \\ ${ }^{1}$ Kurt-Schwabe-Institut für Mess- und Sensortechnik e.V. Meinsberg
}

Kurt-Schwabe-Straße 4, D-04736 Waldheim, www.ksi-meinsberg.de

${ }^{2}$ TU Berlin, Fachgebiet Bioverfahrenstechnik, Institut für Biotechnologie,

Ackerstraße 71-76 ACK 24, D-13355 Berlin

${ }^{3}$ Henze-Hauck Prozessmesstechnik / Analytik GmbH Dünnhauptstraße 14, D-06847 Dessau

\begin{abstract}
Zusammenfassung
Vorgestellt werden prozesstaugliche elektrochemische Sensoren für den Online-Einsatz in der Brauerei. Die unmittelbar zu erfassenden Messgrößen sind der pH-Wert, das Redoxpotential, die Temperatur und die Konzentration von gelöstem Sauerstoff und Kohlendioxid. Entwickelt wurden eine All-solidstate-Kombinationssonde, die mit einer neuartigen Feststoff-Referenzelektrode ausgestattet ist und eine Multisensorsonde mit miniaturisierten elektrochemischen Elektroden.
\end{abstract}

Schlagwörter: elektrochemische Sensoren, All-solid-state-pH- und Referenzelektrode, Multisensorsonde, miniaturisierte Sensoren

\section{Einleitung}

Die Forderung nach lückenloser Kontrolle von Nahrungsgütern vom Erzeuger bis zum Verkauf an den Endverbraucher gewinnt zunehmend an Bedeutung. Um hohe Ausbeuten zu erzielen, gleichbleibende Qualität zu gewährleisten sowie Schadensfälle durch Unregelmäßigkeiten in der Produktion sowie auch durch Umweltschadstoffe und Rückstände in Lebensmitteln zu vermeiden, ist es erforderlich, die Produktqualität während des Herstellungsprozesses online zu kontrollieren.

Für ein solches produktionsbegleitendes Monitoring sind langzeitstabile Sensoren erforder- lich, die unter den speziellen anlagentypischen Bedingungen, z.B. bei den verschiedenen Prozessschritten der Bierherstellung, besonders hohe Anforderungen erfüllen. Dies schließt einen robusten mechanischen Aufbau, eine hohe Druck- und Temperaturbeständigkeit und eine hohe Langzeitstabilität der Sensorsignale ein.

In dieser Präsentation werden zwei verschiedene Messsysteme vorgestellt. Zum einen handelt es sich um eine All-solid-state-Kombinationssonde und zum anderen um eine Multisensorsonde, die mit verschiedenen miniaturisierten elektrochemischen Sensoren bestückt ist. 


\section{All-solid-state-Kombinationssonde Referenzelektrode}

Eine wesentliche, unverzichtbare Komponente nahezu aller elektrochemischen Sensoren und Messeinrichtungen ist die Referenzelektrode. Während die Indikatorelektrode möglichst sensitiv und selektiv auf Änderungen der jeweiligen Inhaltstoffe des Messmediums reagieren sollte, muss die Referenzelektrode ein definiertes, stabiles Potential aufweisen, das von diesen Veränderungen nicht beeinflusst werden darf.

Es erfolgte die Konstruktion einer neuartigen, in ein Mehrloch-Keramikröhrchen eingebetteten Keramik-Referenzelektrode. Das 4-Loch-Keramikrohr mit dem Außendurchmesser $12 \mathrm{~mm}$ besteht aus einer $85 \%$-igen $\mathrm{Al}_{2} \mathrm{O}_{3}$-Keramik (Dimulit C 610) der Firma CeramTec (Bild 1). In zwei der Öffnungen wurde eine poröse Keramik, die als Diaphragma fungiert, mit einem speziellen Kleber eingeklebt. Als innere Ableitung dient ein in erstarrtem $\mathrm{KCl}$ eingebetteter, mit einem Silberdraht kontaktierter $\mathrm{Ag} / \mathrm{AgCl}$ Schmelzkörper. Zum Schmelzen des $\mathrm{KCl}$ bei der Temperatur $780{ }^{\circ} \mathrm{C}$ wurde ein kleiner Ofen (Eigenbau der Henze-Hauck Prozessmesstechnik/Analytik $\mathrm{GmbH}$ ) verwendet [1]. Zur Ausbildung eines konstanten Potentials ist eine Formierung der Feststoff-Referenzelektrode in $3 \mathrm{M}$ $\mathrm{KCl}$-Lösung über 24 Stunden notwendig.
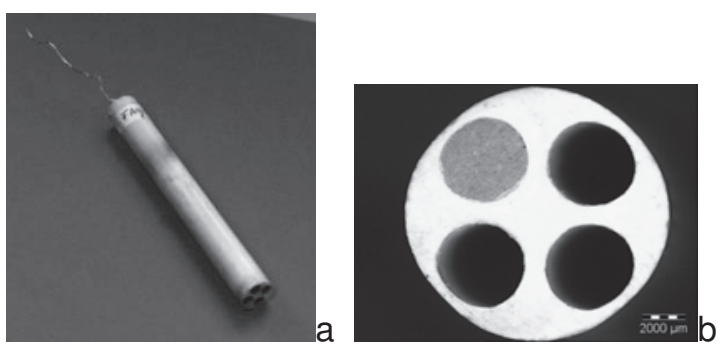

Bild 1. Referenzelektrode mit fest eingeklebtem Keramikdiaphragma

a) Mehrlochkeramikrohr b) Sensorkopf

\section{Indikatorelektroden}

Zur Schaffung einer Kombinationssonde für die Bestimmung des pH-Wertes, des Redoxpotentials, zur amperometrischen Messung der Sauerstoffkonzentration und zur Temperaturüberwachung wurden weitere Elektroden in den anderen Öffnungen des Keramikrohres platziert (Bild 2).

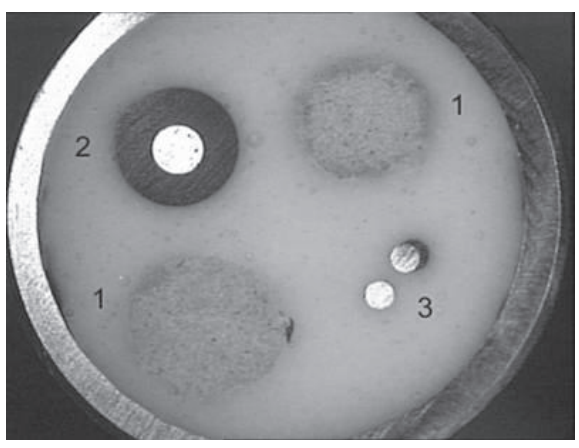

Bild 2. Sensorkopf: 1- Referenzelektroden, 2- Antimonelektrode, 3-Platinelektroden

Die $\mathrm{pH}$-Elektrode besteht aus Antimon, die durch Verpressen von Antimonpulver in einer zylinderförmigen Stahlgussform hergestellt wird. Ihr Durchmesser beträgt ca. $3 \mathrm{~mm}$. Bild 3 zeigt die Ansprechzeit der Antimonelektrode bei Lösungswechsel. Sie beträgt ca. $30 \mathrm{~s}$.

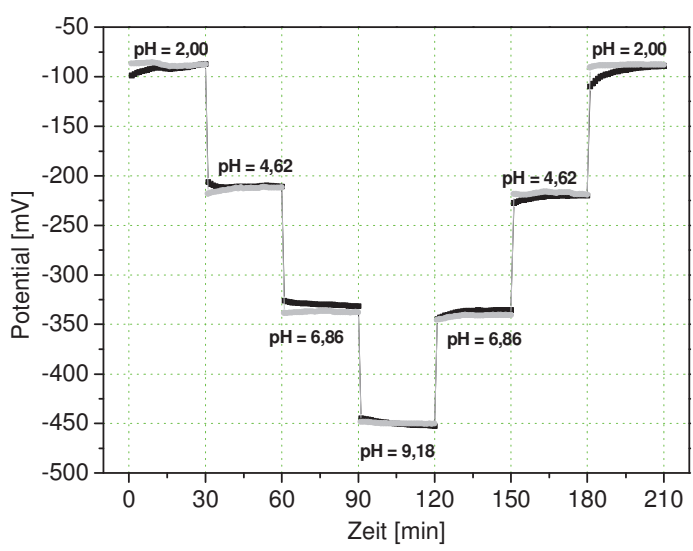

Bild 3. Zeitliches Einstellverhalten zweier $\mathrm{pH}$ Elektroden bei Wechsel in verschiedene Pufferlösungen

Die Messung der Sauerstoffkonzentration erfolgt mit einem offenen amperometrischen Sensor mit Platinelektroden als Arbeits- und Gegenelektrode, die einen Durchmesser von $0,8 \mathrm{~mm}$ haben. Mit Hilfe der Differenzial-PulseVoltammetrie (DPV) und der Square-WaveVoltammetrie (SWV) konnte in Vorversuchen bei Messungen in Bier ein Reduktionspeak im Bereich um $-680 \mathrm{mV}$ beobachtet werden, der eine Abhängigkeit von der Sauerstoffkonzentration zeigt. Vorteile bietet die Methode vor allem bei Untersuchungen im Durchfluss mit stationären Elektroden für Anwendungen in der Prozesskontrolle und für kontinuierliche Messungen, da die Pulse bei der Square-WaveVoltammetrie nur kurzzeitig überlagert werden, ansonsten aber die Anfangsspannung an den Elektroden anliegt. Somit können verschiedene Reaktionsprodukte, die z.B. im Bier vorhanden sind und ebenfalls reduziert werden, durch den Spannungswechsel von der Elektrodenoberfläche wieder entfernt und somit Verschmutzun- 
gen vermieden werden. Die Platinelektroden können gleichfalls zur Messung des Redoxpotentials, der Leitfähigkeit und zur Durchführung voltammetrischer Messungen eingesetzt werden. Ein Temperaturfühler (PT 100) dient zur Temperaturüberwachung.

\section{Messsonde}

Das Mehrlochkeramikrohr mit den eingebauten Elektroden wird in einen aus Edelstahl gefertigten Sensorschaft eingebracht, in dem ebenfalls ein Messwandler zur Digitalisierung der Daten integriert ist (Bild 4).

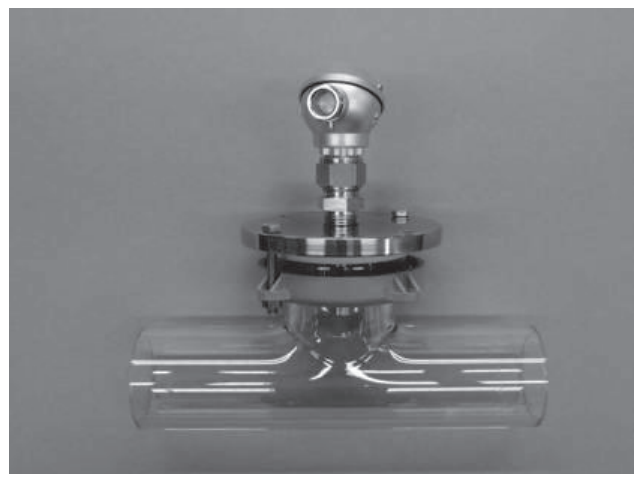

Bild 4. Elektrochemischer Multisensor für die Online Prozesskontrolle

Die Sonde zeichnet sich durch einen robusten mechanischen Aufbau, hohe Druckbeständigkeit und das Fehlen flüssiger Systemkomponenten aus. Der Einsatz des Sensors kann direkt in der Flüssigphase oder in durchströmten Bypässen erfolgen. Gegen Reinigungs- und Desinfektionsmittel, die in der Brauerei verwendet werden, ist die Messsonde beständig.

\section{Multisensorsonde}

Mit Hilfe des Multisensormoduls (Bild 5) können die Parameter $\mathrm{pH}-$ Wert, Redoxpotential, Temperatur, $\mathrm{dCO}_{2}-$ sowie $\mathrm{dO}_{2}$-Konzentration online detektiert werden [2, 3].
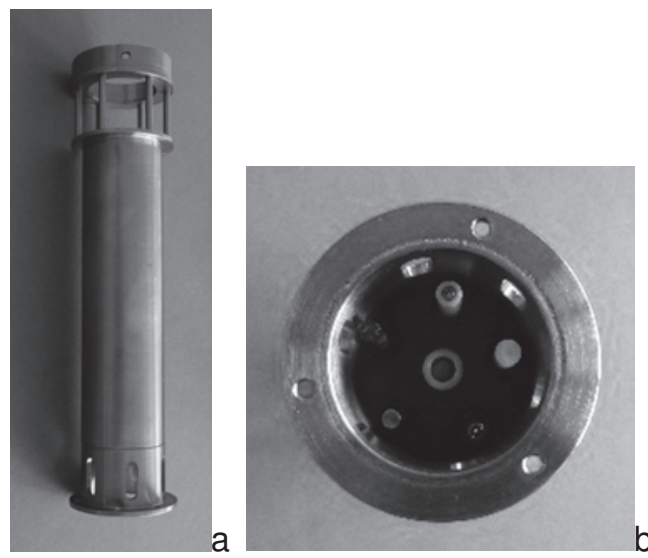

Bild 5. Multisensorsonde

a) Komplettansicht

\section{b) Sicht auf die in der Sonde integrierten Elektroden}

Die Elektroden sind in einem sterilisierbaren Edelstahlgehäuse integriert, so dass die Sonde direkt in der Flüssigphase von Fermentern eingesetzt werden kann. Zusätzlich werden die miniaturisierten Elektroden durch eine Edelstahlkappe vor mechanischer Einwirkung geschützt. Die geschirmten Messkabel werden aus der Sonde herausgeführt und außerhalb des Fermenters mit dem Messgerät verbunden.

In Bild 6 sind die in der Edelstahlsonde integrierten Elektroden dargestellt.

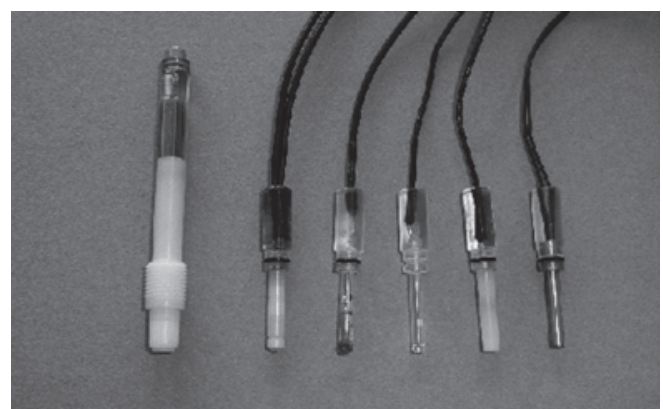

Bild 6. Miniaturisierte Elektroden (v.l.n.r.: dCO $\mathrm{CO}_{2}$, $\mathrm{dO}_{2}$-Sensor, $\mathrm{pH}$-, Redox-, Referenzelektrode, Temperaturfühler)

Bei der pH-Elektrode handelt es sich um eine Glaselektrode, bei der Redoxelektrode um einen in Glas eingeschmolzenen Platindraht. Für die Messung von $\mathrm{pH}-$ Wert und Redoxpotential wird eine separate miniaturisierte Flüssigelektrolyt-Referenzelektrode mit einer $3 \mathrm{M} \mathrm{KCl-Lö-}$ sung als Innenelektrolyt verwendet. Die Sensoren zur Bestimmung des Sauerstoff- und Kohlendioxidgehaltes im Messmedium sind mit gaspermeablen Membranen versehen, um Querempfindlichkeiten gegenüber Bestandteilen der Untersuchungslösung zu vermeiden. Zur Temperaturmessung wird ein Pt1000-Fühler eingesetzt.

Vorteil des Multisensormoduls ist es, dass die miniaturisierten Elektroden einzeln ausgetauscht, aber dennoch wasserdicht in die Sonde eingebaut werden können.

In Bild 7 ist der Zusammenhang zwischen $\mathrm{pH}$ Wert, Sauerstoffgehalt und Redoxpotential in einem braurelevanten Medium (Labormaßstab), bestehend aus Hefezellen und Würzlösung, dargestellt. Das Medium wurde nicht gerührt, da auch in der Praxis die Medien in Braureaktoren in der Regel nicht durchmischt werden. 


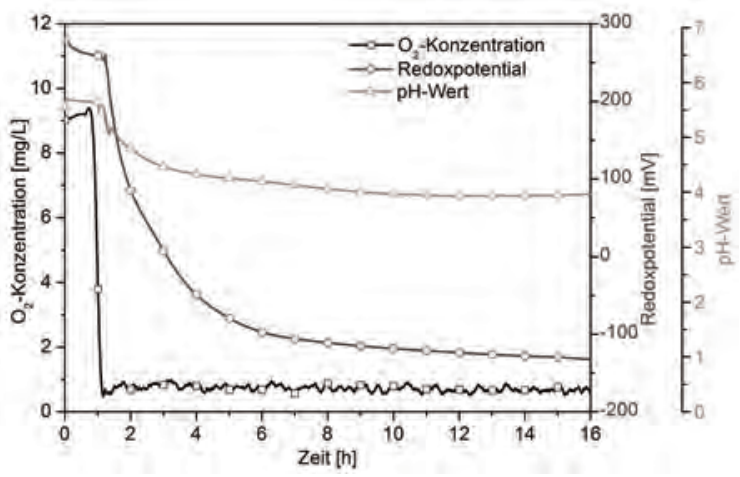

Bild 7. Verlauf von Redoxpotential, $\mathrm{pH}$-Wert und Sauerstoffkonzentration in einem braurelevanten Medium (Raumtemperatur, ungerührt)

Der Stoffwechsel der Hefezellen spiegelt sich in den in Bild 7 dargestellten Kurven wider. Der Sauerstoffgehalt im Messmedium sinkt infolge des Sauerstoffverbrauchs durch die Mikroorganismen zu Beginn der Messung schnell bis unter einen Wert von $1 \mathrm{mg} / \mathrm{L}$. Das Redoxpotential weist ebenfalls in den ersten Stunden eine deutliche Abnahme um mehr als $300 \mathrm{mV}$ auf. Dies steht zum einen im Zusammenhang mit dem Sauerstoffverbrauch, zum anderen auch mit der Umsetzung der vorliegenden Nährstoffe durch die Hefezellen. Der pH-Wert der Untersuchungslösung liegt zu Beginn bei etwa 5,5, sinkt aber innerhalb weniger Stunden auf $\mathrm{pH}=4 \mathrm{ab}$. Ursache dafür ist das von den Hefezellen produzierte Kohlendioxid, welches im Medium teilweise gelöst vorliegt und damit den pH-Wert senkt.

Die Ergebnisse einer Messung in einem LaborGärreaktor sind in Bild 8 dargestellt. Dabei sind die ersten Stunden des Gärversuches gezeigt.

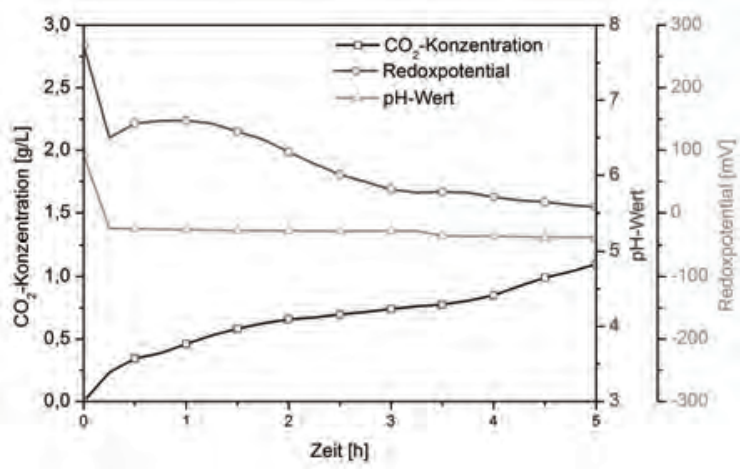

Bild 8. Verlauf von $\mathrm{CO}_{2}$-Konzentration, $\mathrm{pH}$-Wert und Redoxpotential in einem Gärreaktor (Labormaßstab, $12{ }^{\circ} \mathrm{C}$, ungerührt)

Bereits in den ersten fünf Stunden der Gärung stellt sich der $\mathrm{pH}$-Wert auf ein gleichbleibendes Niveau ein $(\mathrm{pH}=5,2)$, wohingegen das Redoxpotential um $250 \mathrm{mV}$ abnimmt. Der Kohlendioxidgehalt steigt bereits in der Anfangsphase deutlich an, was auf den Stoffwechsel der Hefezellen zurückzuführen ist.
Als kritisch wurden die Elektroden mit Membranen betrachtet, da in biologischen Medien die Gefahr besteht, dass sich auf diesen bevorzugt ein Biofilm bildet, der die Messungen verfälschen könnte. Das ist bisher jedoch nicht festgestellt worden. Die mit einer porösen Membran ausgestattete miniaturisierte Referenzelektrode befindet sich bereits seit mehr als 6 Monaten in der Sonde. Lediglich der Innenelektrolyt ist bei der Überprüfung der Sensorik vorsorglich ausgetauscht worden. Das Potential der Referenzelektrode veränderte sich im Vergleich zu dem Potential einer gesättigten $\mathrm{Ag} / \mathrm{AgCl}$-Referenzelektrode nur um wenige $\mathrm{mV}$. Diese Änderung ist demzufolge vernachlässigbar bzw. wird durch regelmäßige Kalibrierung berücksichtigt.

In Bild 9 sind zwei Kalibriergeraden der miniaturisierten $\mathrm{pH}$-Elektrode, aufgenommen im Abstand von 101 Tagen, dargestellt.

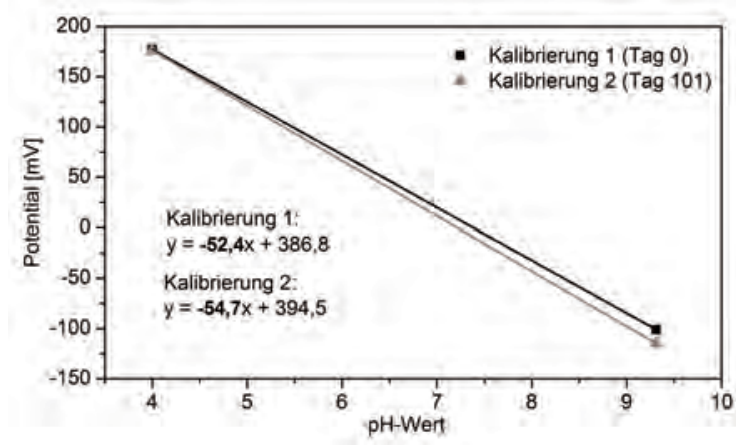

Bild 9. Vergleich der Kalibriergeraden (bei $12^{\circ} \mathrm{C}$ ) der miniaturisierten $\mathrm{pH}$-Elektrode (gegen die miniaturisierte Referenzelektrode) vor Einsatzbeginn und nach 101 Tagen

Die pH-Elektrode weist auch nach 101 Tagen, in denen sie, integriert in der Sonde, mehrfach in verschiedenen Braureaktoren eingesetzt wurde, eine sehr gute Steilheit von $54,7 \mathrm{mV} / \mathrm{pH}$ auf. Diese Elektrode kann demzufolge in weiteren Gärversuchen verwendet werden.

\section{Zusammenfassung}

Die Anwendungen der Sensormodule liegen vor allem im Bereich der biotechnologischen Prozessüberwachung, speziell für den Einsatz bei Gärprozessen in Brauereien und Biogasanlagen, direkt in der Flüssigphase oder in durchströmenden Bypässen.

Die All-solid-state-Kombinationssonde wurde erfolgreich in einer Brauerei direkt im Produktionsprozess der Bierherstellung getestet.

Die Multisensorsonde wurde durch den Kooperationspartner in verschiedenen Laborbraureak- 
toren, aber auch bereits in einem Braureaktor einer Berliner Brauerei eingesetzt und erprobt.

\section{Danksagung}

Teile der Arbeiten wurden gefördert vom Bundesministerium für Wirtschaft und Technologie (BMWi) über die Arbeitsgemeinschaft industrieller Forschungsvereinigung „Otto von Guericke“ e.V. (AiF) im Rahmen des Programms „Zentrales Innovationsprogramm Mittelstand“ (ZIM)Kooperationen unter dem Förderkennzeichen KF 22118304PR9 und durch die Kurt-SchwabeStiftung mit einem Kurt-Schwabe-Stipendium.

\section{Literatur}

[1] W. Vonau, U. Enseleit, W. Oelßner, J. Henze: Inline Prozesskontrolle. $\mathrm{pH}$-Elektrode für den Einsatz bei der Bierherstellung, GIT LaborFachzeitschrift 56 (2012) 2, 105-107

[2] M. Bertau, W. Vonau, S. Herrmann, S. Päßler: Sensorgestütztes Mikroreaktorsystem, DE 10 2009048113 A1 (Patent)

[3] A. Bockisch, J. Biering, T. Tyrell, S. Päßler, W. Vonau, P. Neubauer, S. Junne: Oscillating Conditions in Yeast Fermentation: Multiposition Sensors and Scale Down Experiments (Poster), Tagung "Effiziente Verfahren und Anlagen in der Bioverfahrenstechnik und Lebensmittelbiotechnologie", 06.-08. Mai 2013, Bad Wildungen 